\title{
Impact of mycolactone produced by Mycobacterium ulcerans on life-history traits of Aedes aegypti (L.) and resulting habitat selection for oviposition
}

\author{
Mashlawi, A.M. ${ }^{1,4^{*}}$, Jordan, H.R. ${ }^{2}$, Crippen, T.L. ${ }^{3}$ and Tomberlin, J.K. ${ }^{1}$ \\ ${ }^{1}$ Department of Entomology, Texas A\&M University, College Station, TX, USA \\ ${ }^{2}$ Mississippi State University, Starkville, MS, USA \\ ${ }^{3}$ Agricultural Research Service, USDA, College Station, TX, USA \\ ${ }^{4}$ Biology Department, Faculty of Science, Jazan University, Jazan, Saudi Arabia \\ *Corresponding author e-mail: abadimashlawi@gmail.com \\ Received 8 July 2020; received in revised form 14 September 2020; accepted 17 September 2020
}

\begin{abstract}
Buruli ulcer (BU) is a globally recognized, yet largely neglected tropical disease whose etiologic agent is Mycobacterium ulcerans. Although the exact mode of transmission is unclear, epidemiological evidence links BU incidence with slow-moving or stagnant, aquatic habitats, and laboratory-based experiments have shown disease manifestation in animals with dermal punctures. Therefore, hypotheses for transmission include contact with slowmoving aquatic habitats and associated biting aquatic insects, such as mosquitoes. Recent research demonstrated the toxin produced by $M$. ulcerans, mycolactone, is an attractant for adult mosquitoes seeking a blood-meal as well as oviposition sites. In the study presented here, we examined the impact of mycolactone at different concentrations on immature lifehistory traits of Aedes aegypti, which commonly occurs in the same environment as $M$. ulcerans. We determined percent egg hatch was not significantly different across treatments. However, concentration impacted the survivorship of larval mosquitoes to the adult stage $(p<0.001)$. Resulting adults also showed a slight preference, but not significant $(p>0.05)$, for oviposition in habitats contaminated with mycolactone suggesting a legacy effect.
\end{abstract}

\section{INTRODUCTION}

BURULI ULCER (BU) is a globally recognized, yet highly neglected tropical disease caused by Mycobacterium ulcerans (Merritt et al., 2010). Buruli ulcer is the third most common mycobacterial disease of humans after tuberculosis and leprosy (Vincent et al., 2014) mostly affecting individuals between the ages of 4 to 15 years old (Vincent et al., 2014; Williamson et al., 2008). The disease is prevalent in at least 33 countries, with most cases occurring in West Africa (Williamson et al., 2008; Merritt et al., 2010).

Mycobacterium ulcerans produces mycolactone, a cytotoxic and immunosuppressive polyketide-derived macrolide responsible for skin ulcerations, which are the primary $\mathrm{BU}$ disease manifestations (Mve-Obiang et al., 2003). The ulcers are painless; however, if not treated, they can expand and result in secondary infection, bone deformation, and osteomyelitis (Vincent $e t$ $a l ., 2014)$. Though mortality is low, there is significant associated morbidity leading to socioeconomic burden and social stigma (Yeboah-Manu et al., 2013).

The exact mode of transmission of $M$. ulcerans is not known. Recent work using animal models has shown a dermal puncture is necessary to establish infection and BU disease pathology (Williamson et al., 2014). Epidemiological evidence links BU incidence with slow-moving aquatic habitats, especially in areas prone to anthropogenic disturbance and flooding. This evidence has been strengthened by the finding of 
M. ulcerans DNA in cisterns and wells within villages (Merritt et al., 2010; Williamson et al., 2012, 2014; Wallace et al., 2017). Furthermore, insects, such as black flies (Diptera: Simuliidae), mosquitoes (Diptera: Culicidae) (Merritt et al., 2010), March flies (Diptera: Tabanidae), and sand flies (Diptera: Ceratopogonidae) (Quek et al., 2007), associated with one or the other of these environments (i.e., mosquitoes in ponds or cisterns) have been suggested as potential vectors. Moreover, $M$. ulcerans DNA has been identified in aquatic insects (Naucoridae and Belostomatidae) obtained from endemic areas in Africa (Williamson et al., 2008). In fact, under laboratory conditions, $M$. ulcerans was transmitted to mice by the bite of $M$. ulcerans infected aquatic hemipterans (Naucoridae) (Marsollier et al., 2002). Additionally, the mosquito Aedes notoscriptus, (Skuse) (Diptera: Culicidae) was able to mechanically produce infection in 2 of 11 mice whose tails had been dipped in $M$. ulcerans culture (Wallace et al., 2017).

A recent study in our laboratories demonstrated mycolactone influences behavior of the yellow fever mosquito, Aedes aegypti aegypti, (L.) (Diptera: Culicidae) (Sanders et al., 2017), which occurs in similar environments (Garnham et al., 1946; Christophers, 1960) as M. ulcerans (Williamson et al., 2012). In the study, 29\% more were attracted to blood-feeders treated with $1.0 \mu \mathrm{g} / \mathrm{ml}$ mycolactone compared to control feeders. They further demonstrated naïve mosquitoes (never exposed to $M$. ulcerans or mycolactone) preferred to oviposit (64\%) in areas containing mycolactone at the same concentration $(1.0 \mu \mathrm{g} / \mathrm{ml})$ that enhanced attraction to the blood-feeders (Sanders et al., 2017). However, the impact of mycolactone on subsequent mosquito development and resulting adult behavior is not known.

Since many mosquitoes (e.g., Ae. aegypti) have strong oviposition preferences for sites with bacteria (Ponnusamy et al., 2008), the relationship between $\mathrm{BU}$ and Ae. aegypti may become even more complex if Ae. aegypti prefer to oviposit in sites similar to their larval habitat. In fact, volatile organic compounds released by bacteria have been demonstrated to drive mosquito preferences to oviposit in bacteria-present vs. bacteria-absent sites (Corbet, 1985; Albeny-Simoes et al., 2014). A potential source of these volatiles is the by-products produced by bacterial quorum sensing (QS) or other secondary metabolism, which are modulators of inter-kingdom behavior, especially insects (Tomberlin et al., 2016).

Quorum sensing is a process coordinating gene expression according to the density of a bacterial population (Miller \& Bassler, 2001). This process allows for population-level physiological and other phenotypical shifts by bacteria, such as biofilm formation or virulence expression (Miller \& Bassler, 2001). A recent laboratory study demonstrated QS by the human dermal commensal, Staphylococcus epidermidis enhanced mosquito attraction to a bloodfeeders by $74 \%$ versus the strain unable to QS (Zhang et al., 2015).

The macrolide structure of mycolactone suggests the possibility that the molecule may be an antagonist to bacteria with QS machinery (similar to acyl-homoserine lactones) or may serve as a regulator of secondary metabolism (Romero et al., 2011). And, as previously stated, mycolactone is a known mosquito attractant (Sanders et al., 2016). Based on these and previously published data, we sought in the current study to determine whether: 1) immature $A e$. aegypti development and survivorship in the presence of mycolactone were dosedependent, and if so, 2) do resulting $A e$. aegypti adults prefer to oviposit in habitats containing mycloactone at rates similar to those experienced during larval development.

\section{MATERIALS AND METHODS}

\section{Insect Colonies}

Aedes aegypti (Liverpool strain) was used in this study due to the common occurrence of this species in areas endemic to $M$. ulcerans as mentioned in the introduction. Aedes aegypti was maintained in a colony housed in a walk-in growth chamber at $25.0^{\circ} \mathrm{C} \pm 2.5^{\circ} \mathrm{C}$, 12:12 L:D, and $70.0 \% \pm 5.0 \% \mathrm{RH}$ at the Forensic Laboratory for Investigative Entomological 
Sciences (FLIES) Facility (Texas A\&M University, College Station, TX, USA). Eggs were placed in $1 \mathrm{~L}$ of distilled water held in containers $(17.5 \mathrm{~cm} \times 12 \mathrm{~cm} \times 4.5 \mathrm{~cm})$ at room temperature. Resulting larvae were separated into similar containers at a density of 100-200 larvae/L. Larvae were provided fish food ad libitum (TetraMin diet by Tetra Blacksburg, VA, USA) to avoid overfeeding. Distilled water was added to the containers as needed; containers were checked every $12 \mathrm{~h}$ for the presence of pupae, which were then partitioned into $60 \mathrm{ml}$ cups (containing $40 \mathrm{ml}$ of distilled water) at a density of 50 pupae/cup. These cups were placed individually inside an insect cage $(30 \times 30 \times$ $30 \mathrm{~cm}$ ), and pupae were monitored for adult emergence. Newly emerged adults were provided with a $10 \%$ sucrose solution via a wet cotton ball placed on top of the cage.

\section{Mycolactone}

Mycolactone at $1.0 \mu \mathrm{g} / \mathrm{ml}, 0.5 \mu \mathrm{g} / \mathrm{ml}$, and $0.05 \mu \mathrm{g} / \mathrm{ml}$ concentrations were used in this study. Mycolactone was prepared using previously described methods (Adusumilli et al., 2005) with slight modifications; specifically, $M$. ulcerans Agy99 was grown on M7H10 plates. Bacteria were scraped from the plates, dried and weighed. Mycolactone was isolated from acetone soluble lipids (ASLs) using centripetal chromatography by running concentrated lipids (dried down ASLs resuspended in $2 \mathrm{ml}$ 96:4 dichloromethane: $\mathrm{MeOH})$ through a chromatotron to separate individual lipid species using 96:4 dichloromethane: MeOH followed by 90:10 chloroform: $\mathrm{MeOH}$ with fraction collection and visualization of the UV-active species and further fractionation and collection. Fractions were analyzed by TLC ran in 90:10:1 chloroform:methanol:water solvent using a mycolactone control and visualized with ceric sulfate-ammonium molybdate in $2 \mathrm{M}$ sulfuric acid stain with Rf values compared against a mycolactone control. A cytopathicity assay was conducted to confirm activity as previously described (Mve-Obiang et al., 2003). Mycolactone concentration was extrapolated from $M$. ulcerans cell weight and corresponding colony count, where one cell was estimated to produce approximately
$1 \mathrm{pg}$ of mycolactone. This information was used along with the qPCR values of $M$. ulcerans from environmental samples to choose mycolactone concentrations used in this study, with an effort to represent a range of detected concentrations corresponding to qPCR-assayed environmental matrices collected from Ghana and Benin (Williamson et al., 2012) [HRJ, unpublished data]. Prepared mycolactone was solubilized with 95\% ethanol and stored in amber vials placed in the dark at room temperature to prevent degradation due to ultraviolet light (Marion et al., 2012).

\section{Larval Growth/Survivorship Assay}

All experiments were conducted under the laboratory conditions previously described (Sanders et al., 2017). For the larval growth/ survivorship assay, 40 Ae. aegypti eggs total, taken from multiple females from the colony, were placed in a round glass jar (236 ml) (Packaging Options Direct, Louis, MO, USA) containing $55 \mathrm{ml}$ of distilled water. This density was selected based on preliminary experiments where the greatest level of survivorship to the adult stage was determined. Approximately 30-50 mg Tetramin fish food was placed in the water at the time the eggs were introduced. Larvae were reared as previously described.

For the treatments, $50 \mu \mathrm{l}$ mycolactone was applied using micropipette (Eppendorf, $\mathrm{NY}, \mathrm{USA}$ ) at $1.0 \mu \mathrm{g} / \mathrm{ml}$ (high), $0.5 \mu \mathrm{g} / \mathrm{ml}$ (medium), and $0.05 \mu \mathrm{g} / \mathrm{ml}$ (low) concentration with $95 \%$ ethanol serving as the solvent. Ethanol $(50 \mu \mathrm{l})$ alone served as the positive control; a negative control (nothing applied) was also used. Jars with three concentrations $(1.0 \mu \mathrm{g} / \mathrm{ml}, 0.5 \mu \mathrm{g} / \mathrm{ml}$ and 0.05 $\mu \mathrm{g} / \mathrm{ml}$ ) were used as the containers for each replicate during the experiment. Each mycolactone treatment was added to a glass jar containing distilled water $(55 \mathrm{ml})$ and allowed to ventilate for $30 \mathrm{~min}$ prior to the introduction of mosquito eggs. Glass jars (replicates) were then placed individually in a mosquito-breeder (L: $21 \mathrm{~cm} \times \mathrm{W}: 12 \mathrm{~cm}$ ) (BioQuip, CA, USA). To avoid sampling bias, replicates were checked every $12 \mathrm{~h}$ for pupae and adult emergence (total percent recorded). The sex of each emerged adult was 
also recorded, and the percentage hatch rate and larval survivorship to adulthood were measured. Four trials of the experiment were completed.

\section{Oviposition assay}

Based on results from the larval growth/ survivorship assay, mosquitoes were reared in the presence of mycolactone at $0.5 \mu \mathrm{g} / \mathrm{ml}$ ethanol as it yielded the most similar adult emergence patterns to those seen in controls when compared to the other treatments. The quantity of mycolactone/solvent (50 $\mu \mathrm{l})$ applied was adjusted for the volume of water within a container. Cluster eggs were placed in $1 \mathrm{~L}$ of distilled water treated with either $0.5 \mu \mathrm{g}$ mycolactone/ml ethanol, just ethanol or a negative control (nothing applied) as control, and held in $30 \mathrm{~cm} \times 23 \mathrm{~cm} \times 5 \mathrm{~cm}$ mosquito pans (Bioquip, CA, USA) in the walk-in incubator at $25.0^{\circ} \mathrm{C} \pm 2.5^{\circ} \mathrm{C}, 12: 12 \mathrm{~L}: \mathrm{D}$, and $70.0 \% \pm 5.0 \% \mathrm{RH}$. Larvae were reared and monitored as described previously until emergence. Adult mosquitoes aged 5-10-dold were then starved for $24 \mathrm{~h}$ prior to being provided a blood-meal using methods previously described (Sanford \& Tomberlin, 2011).

Approximately $72 \mathrm{~h}$ after blood-feeding, three, $150 \mathrm{ml}$ black containers were placed equidistant from one another in triangulated fashion in each cage representing each treatment. In each container, a single filter paper (11 cm in diameter, Whatman No. 1) was provided as an oviposition site as a normal substrate for eggs collection (Imam et al., 2014). Each filter paper, which represented a treatment or control $(0.5 \mu \mathrm{g}$ mycolactone, ethanol, blank) was divided equally, where half of the paper was treated, and the paper was placed in water with the orientation of the treated side of the filter paper-oriented randomly east or west in the cage. Ae. aegypti were allowed to oviposit for $72 \mathrm{~h}$, after which, the filter papers were removed, and dried in the incubator for four days. Eggs present on each half were then tabulated. Three trials of the experiment were completed. For each trial, the location of the treatment within the triangulated positions was rotated as well as the orientation of the treatment east or west.

\section{Statistical Analysis}

An analysis of variance (ANOVA) was used to assess larval growth/survivorship assay data (JMP ${ }^{\circ}$ Pro 12.0.1, Cary, NC, USA). Tukey's multiple comparison procedure and statistical test were used to separate means following a significant $\mathrm{F}$ test. The alpha value was set at $p<0.05$. For the oviposition study, an ANOVA was used to determine differences in the number of eggs deposited on the three treatments present in a given cage. For eggs deposited on half of the filter paper with either mycolactone or ethanol, the probability $(\mathrm{P})$ of response (i.e., oviposition) by Ae. aegypti was examined for a significant difference $(p<0.05)$. Furthermore, a comparison of mosquito responses across doses was conducted with PROC GLIMMIX (SAS University Edition, Version 9.4), a generalized linear mixed model (GLMM). The odds of treatment/blank control (i.e., attraction and oviposition) by Ae. aegypti to the different treatments was examined for significant difference $(p<0.05)$ between mycolactone and ethanol treatments. Replicate was included in the model as a random factor.

\section{RESULTS}

\section{Larval Hatch/Survivorship Assay}

No significant $(\mathrm{F}=0.781 ; \mathrm{df}=4,59 ; p=0.544)$ difference in egg hatch was determined across treatments. Furthermore, no significant $(\mathrm{F}=0.738 ; \mathrm{df}=12,59 ; p=0.707)$ interaction was determined between trial and treatment. Although no difference in response of mosquitoes to treatments was detected (i.e. treatment by trial interaction), a trial effect was determined $(\mathrm{F}=9.099 ; \mathrm{df}=$ $3,59 ; p<0.001)$. However, it should be noted the order of treatment responses was consistent across trials. Trials 1 and 2 were significantly $(p<0.05)$ different from trials 3 and 4 . The average egg hatch in trial one and two was $97.50 \% \pm 0.01 \%$ (Figure 1a) and $80.10 \% \pm 0.03 \%$ (Figure $1 \mathrm{~b}$ ) in trials three and four.

Significant difference $(\mathrm{F}=10.085 ; \mathrm{df}=4$, $53 ; p<0.001)$ across treatments was determined for survivorship from egg to pupal and adult stage. No significant $(\mathrm{F}=1.070 ; \mathrm{df}=12$, 
$53 ; p=0.414)$ interaction was determined between trial and treatment. No trial effect was determined $(\mathrm{F}=1.436 ; \mathrm{df}=3,53 ; p=$ 0.249). The average survival of eggs to the pupal and adult stage for the controls was $81 \%$ or greater. Survival of those exposed to the different mycolactone treatments was greatest for the middle dose $(0.5 \mu \mathrm{g} / \mathrm{ml} ; 58 \%)$, which was not significantly different from the controls; however, survival, when exposed to this treatment, was almost double of what was observed for those assigned the high $(1.0 \mu \mathrm{g} / \mathrm{ml})$ and low $(0.05 \mu \mathrm{g} / \mathrm{ml})$ dose treatments ( $\sim 30-39 \%$ respectively) (Figure 2$)$. No mortality was observed at the pupal stage.

Significant differences $(\mathrm{F}=4.837 ; \mathrm{df}=4$, $52 ; p=0.004$ ) in development time to the pupal stage were determined across treatments. No significant interaction $(\mathrm{F}=1.711$; $\mathrm{df}=12,53 ; p=0.110$ ) was determined

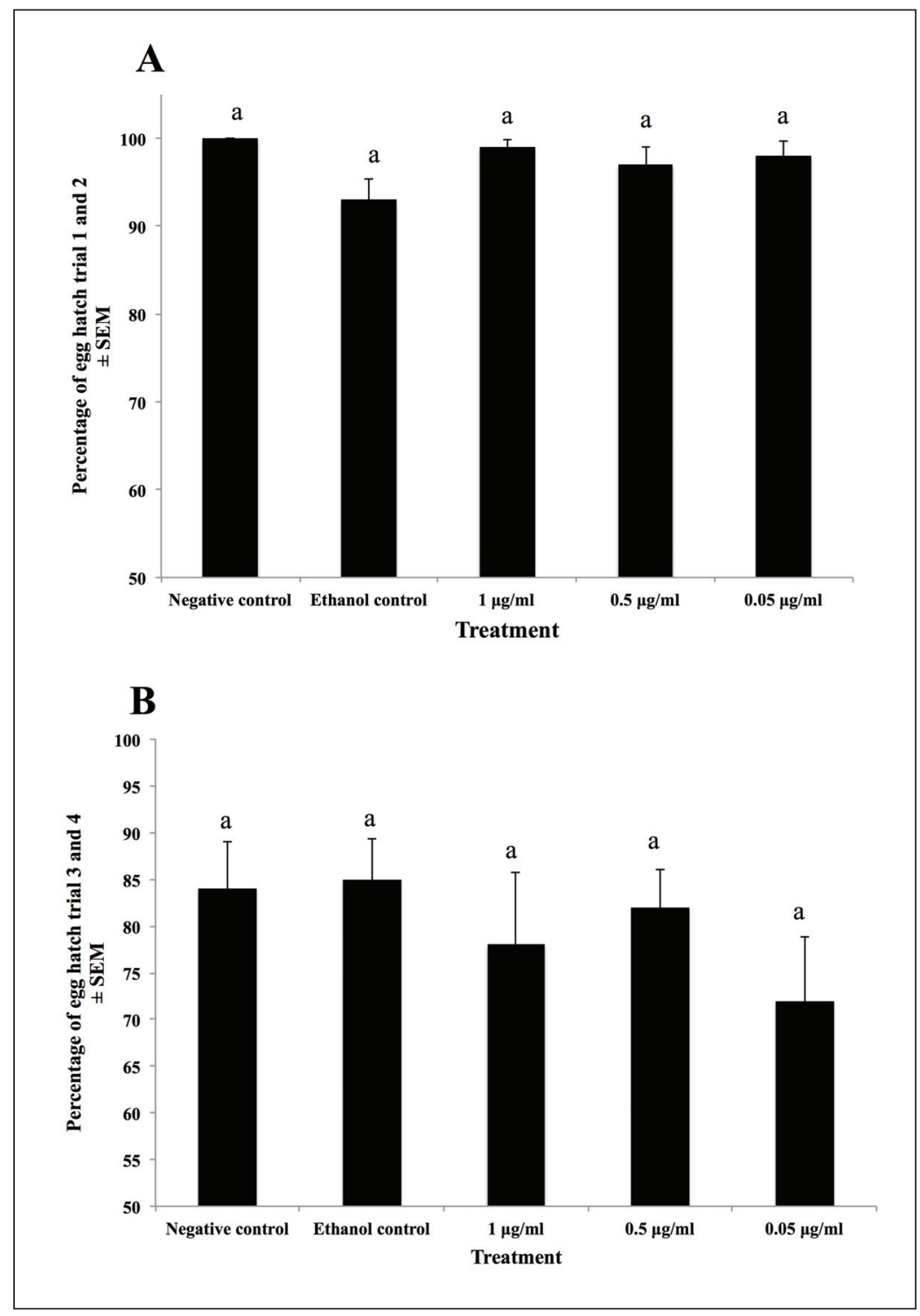

Figure 1. Percentage of Ae. aegypti egg hatch $(\mathrm{n}=4) \pm$ SEM exposed to three concentrations of mycolactone, negative control, or ethanol control at $25.0^{\circ} \mathrm{C}$ $\pm 2.5^{\circ} \mathrm{C}, 12: 12 \mathrm{~L}: \mathrm{D}$, and $70.0 \% \pm 5.0 \% \mathrm{RH}$ for (A) trials 1 and 2 for (B) trials 3 and 4 combined. 


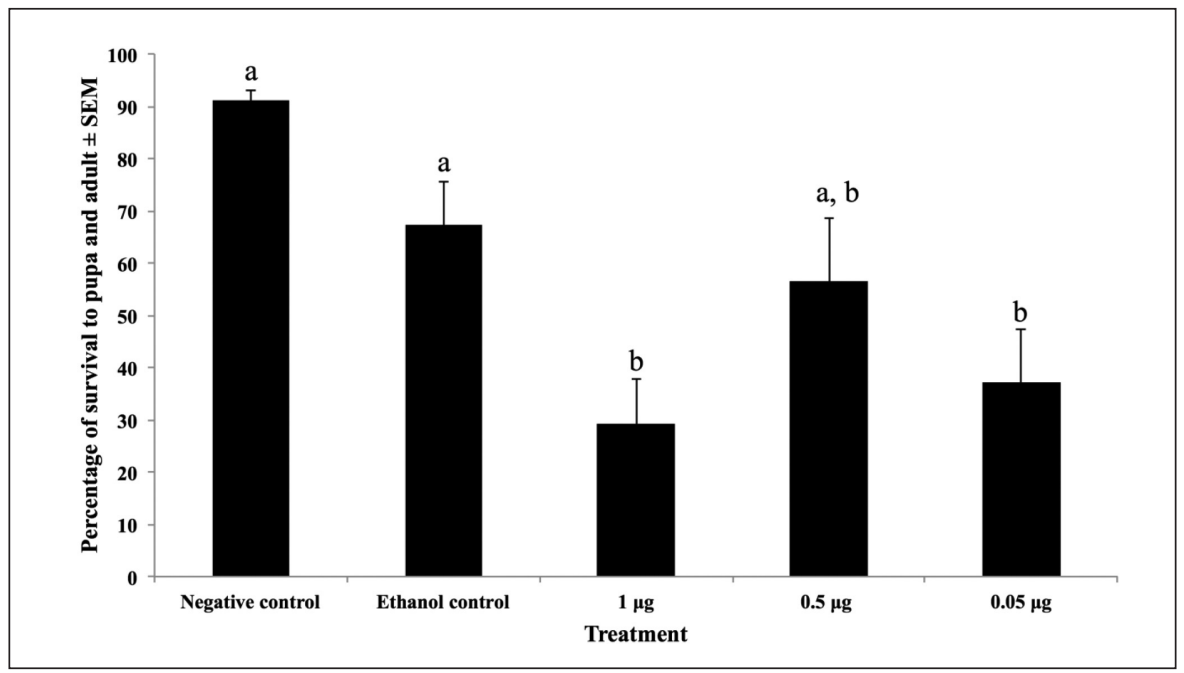

Figure 2. Percentage of Ae. aegypti survival from egg to pupal and adult stages $(\mathrm{n}=4)$ \pm SEM exposed to three concentrations of mycolactone, negative control, or ethanol control at $25.0^{\circ} \mathrm{C} \pm 2.5^{\circ} \mathrm{C}, 12: 12 \mathrm{~L}: \mathrm{D}$, and $70.0 \% \pm 5.0 \% \mathrm{RH}$.

Table 1. Mean \pm SEM of time (d) from egg to pupae and adult of Ae. aegypti exposed to different concentration of mycolactone, as well as negative control and ethanol control at $25.0^{\circ} \pm 2.5^{\circ} \mathrm{C}, 12: 12 \mathrm{~L}: \mathrm{D}$, and $70.0 \% \pm 5.0 \% \mathrm{RH}$

\begin{tabular}{|c|c|c|c|c|}
\hline \multicolumn{5}{|c|}{ Mean time from egg to pupae (d) Mean \pm SEM } \\
\hline Treatment & \multicolumn{2}{|c|}{ Trial 1,3 and 4} & \multicolumn{2}{|c|}{ Trial 2,3 and 4} \\
\hline Negative control* & \multicolumn{2}{|c|}{$7.35 \pm 0.15^{\mathrm{b}}$} & \multicolumn{2}{|c|}{$7.57 \pm 0.06^{\mathrm{a}}$} \\
\hline Ethanol control & \multicolumn{2}{|c|}{$6.87 \pm 0.20^{\mathrm{b}}$} & \multicolumn{2}{|c|}{$7.57 \pm 0.41^{\mathrm{a}}$} \\
\hline $1 \mu \mathrm{g} / \mathrm{ml}$ & \multicolumn{2}{|c|}{$8.07 \pm 0.46^{\mathrm{a}}$} & \multicolumn{2}{|c|}{$8.33 \pm 0.40^{\mathrm{a}}$} \\
\hline $0.5 \mu \mathrm{g} / \mathrm{ml}$ & \multicolumn{2}{|c|}{$7.33 \pm 0.18^{b}$} & \multicolumn{2}{|c|}{$7.58 \pm 0.10^{\mathrm{a}}$} \\
\hline $0.05 \mu \mathrm{g} / \mathrm{ml}$ & \multicolumn{2}{|c|}{$7.48 \pm 0.29^{\mathrm{a}, \mathrm{b}}$} & \multicolumn{2}{|c|}{$7.63 \pm 0.30^{\mathrm{a}}$} \\
\hline \multicolumn{5}{|c|}{ Mean time from egg to adult (d) Mean \pm SEM } \\
\hline Treatment & Trial 1 & \multicolumn{2}{|c|}{ Trial 3} & Trial 2 and 4 \\
\hline Negative control* & $9.53 \pm 0.09^{\mathrm{a}}$ & \multicolumn{2}{|c|}{$13.70 \pm 0.06^{\mathrm{a}}$} & $12.77 \pm 0.05^{\mathrm{a}}$ \\
\hline Ethanol control & $9.55 \pm 0.07^{\mathrm{a}}$ & \multicolumn{2}{|c|}{$13.80 \pm 0.00^{\mathrm{a}}$} & $12.63 \pm 0.08^{\mathrm{a}}$ \\
\hline $1 \mu \mathrm{g} / \mathrm{ml}$ & $9.33 \pm 0.00^{\mathrm{a}, \mathrm{b}}$ & \multicolumn{2}{|c|}{$13.30 \pm 0.30^{\mathrm{a}}$} & $12.48 \pm 0.11^{\mathrm{a}}$ \\
\hline $0.5 \mu \mathrm{g} / \mathrm{ml}$ & $9.24 \pm 0.24^{\mathrm{a}, \mathrm{b}}$ & \multicolumn{2}{|c|}{$13.80 \pm 0.00^{\mathrm{a}}$} & $12.55 \pm 0.13^{\mathrm{a}}$ \\
\hline $0.05 \mu \mathrm{g} / \mathrm{ml}$ & $9.00 \pm 0.00^{\mathrm{b}}$ & \multicolumn{2}{|c|}{$13.80 \pm 0.35^{\mathrm{a}}$} & $12.50 \pm 0.13^{\mathrm{a}}$ \\
\hline
\end{tabular}

*Negative control was no treatment (just water).

between trial and treatment. However, a trial effect was determined $(\mathrm{F}=3.750 ; \mathrm{df}=3$, $53 ; p=0.020)$. Therefore, the results for development time from egg to the pupal stage were grouped by trial. The average time from egg to pupa \pm SEM is shown in (Table 1 ).

For trials 1,3 and 4 , significant $(\mathrm{F}=6.129$; $\mathrm{df}=4,37 ; p=0.002$ ) the difference in development time from egg to the pupal stage was determined across treatments. From these, individuals in the negative control group needed $7.35 \mathrm{~d}$ or less to pupate. The development time for those exposed to the high dose for trials 1,3, and 4 was significantly greater $(\sim 8 \mathrm{~d})$ than for individuals in other treatments ( $\sim 7 \mathrm{~d}$ for the mean of control, low and middle doses). Furthermore, a significant $(\mathrm{F}=2.796 ; \mathrm{df}=8,37 ; p=0.026)$ interaction 
was determined between trial and treatment. For trials 2, 3 and 4, treatment did not significantly ( $\mathrm{F}=1.820 ; \mathrm{df}=4,40 ; p=0.155)$ impact development time from egg to the pupal stage.

Significant difference $(\mathrm{F}=5.332 ; \mathrm{df}=4$, $52 ; p=0.002)$ in development time to the adult stage was determined across treatments (Table 1). No significant ( $\mathrm{F}=0.844$; $\mathrm{df}=12,52 ; p=0.607$ ) interaction was determined between trial and treatment. A trial effect was determined $(\mathrm{F}=760.306$; $\mathrm{df}=3,53 ; p<0.001)$ in development time to the adult.

A significant $(\mathrm{F}=5.586 ; \mathrm{df}=4,11 ; p=$ 0.024 ) difference in development time from egg to the adult stage was determined across treatments in trial 1. Individuals in the ethanol control groups needed a maximum of $9.55 \mathrm{~d}$ to become adults, which was greater than observed for the treatments. The development time for those exposed to the high dose $(1 \mathrm{\mu g} / \mathrm{ml})$ was significantly greater $(\sim 9.33 \mathrm{~d})$ than for individuals in other treatments ( $9.12 \mathrm{~d}$ for mean of ethanol control, low and middle doses). Trial 1 was significantly $(p<0.05)$ different from trials 2 , 3 and 4 , but none of these trials demonstrated a significant (trials 2 and $4: \mathrm{F}=1.486 ; \mathrm{df}=4$, $29 ; p=0.244$; trial 3: $\mathrm{F}=1.910 ; \mathrm{df}=4,10 ; p=$ 0.228 ) impact on development from egg to adult.

\section{Oviposition Assay}

Mosquitoes were reared in containers treated with $0.5 \mu \mathrm{g} / \mathrm{ml}$ mycolactone or reared in the absence of mycolactone (ethanol as control); the number of eggs deposited in sites with the same mycolactone dose or the controls was determined (Figure 3). Mosquitoes reared in the control did not show a significant $(p>0.05)$ preference for such sites when ovipositing. However, mosquitoes reared in the presence of mycolactone were slightly more likely to deposit eggs on the portion of filter paper treated with either mycolactone $\left(p<0.0001, X^{2}=184.81, \log \right.$ odds of responding to mycolactone versus the blank $=0.69 \pm 0.05$, Figure 4 ) or ethanol $\left(p<0.0001, X^{2}=15.65, \log\right.$ odds of responding to ethanol versus the blank $=0.23 \pm 0.06$, Figure 3) relative to the untreated half. In contrast, mosquitoes initially reared in the presence of ethanol appeared to be repelled by the presence of either mycolactone $(p<$ $0.0001, X^{2}=28.97, \log$ odds of responding to

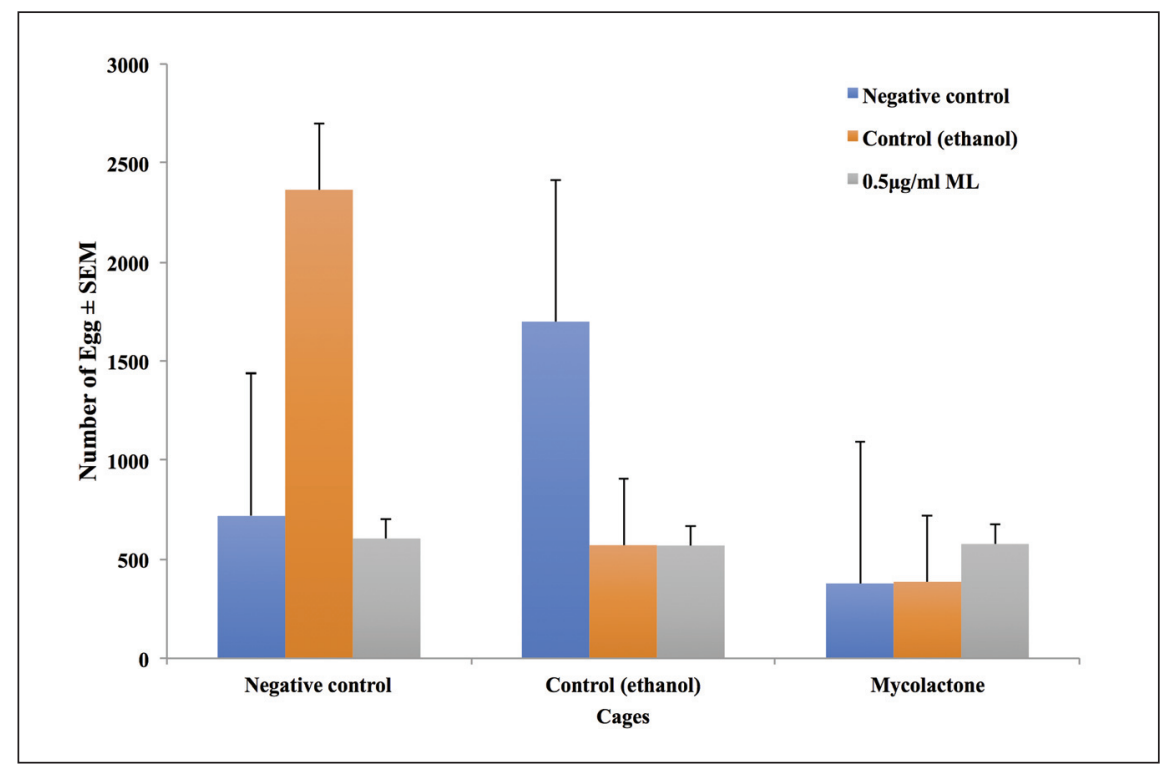

Figure 3. Number of Ae. aegypti eggs laid on filter paper treated with ethanol or mycolactone by adults reared in the presence of 0.5 mycolactone $\mu \mathrm{g} / 1 \mathrm{ml}$ ethanol, 1 $\mathrm{ml}$ ethanol and negative control at $25.0^{\circ} \mathrm{C} \pm 2.5^{\circ} \mathrm{C}, 12: 12 \mathrm{~L}: \mathrm{D}$, and $70.0 \% \pm 5.0 \% \mathrm{RH}$ during larval development. 


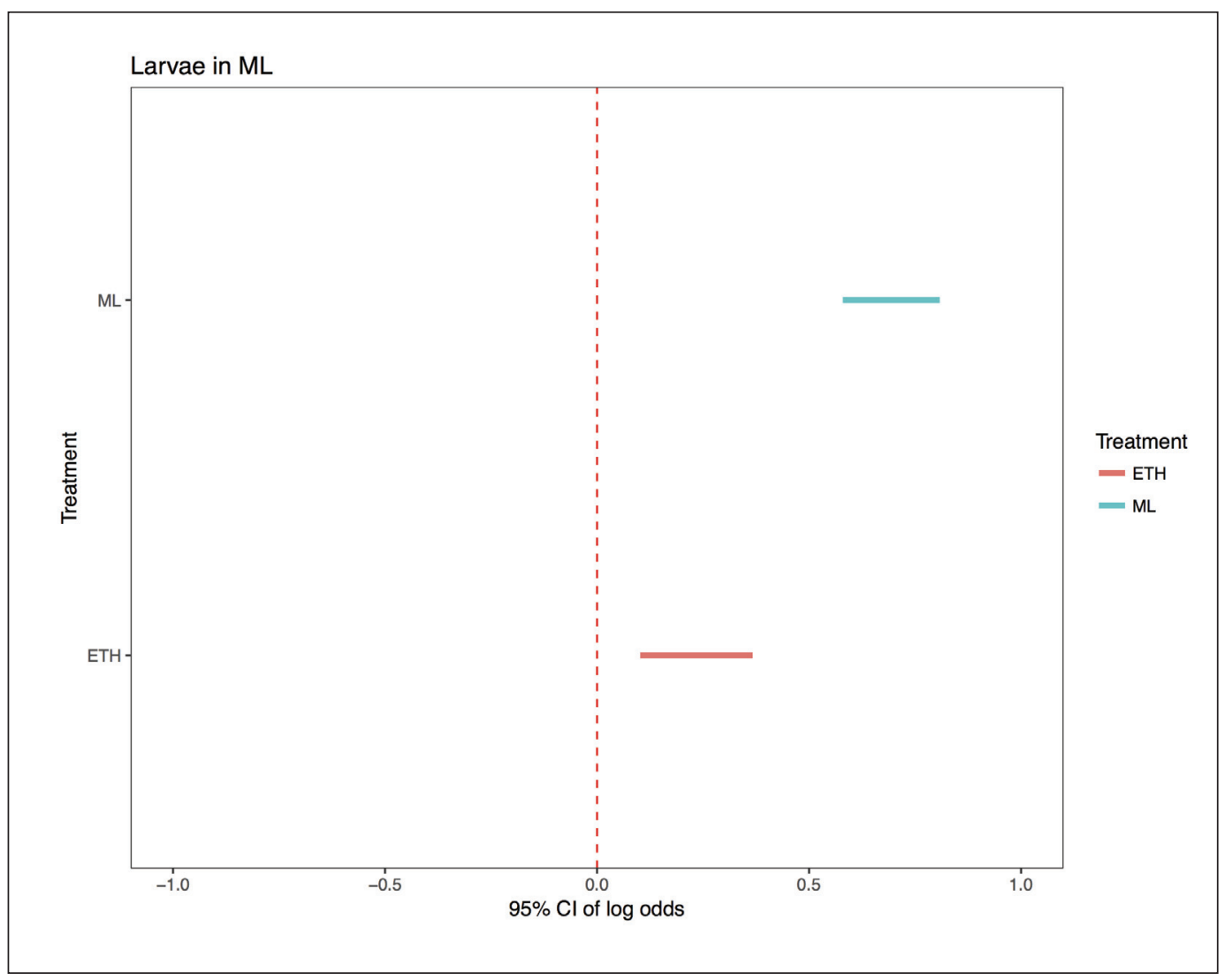

Figure 4. Log odds of Ae. aegypti laying eggs on filter paper treated with $0.5 \mu \mathrm{g}$ mycolactone at $25.0^{\circ} \mathrm{C}$ $\pm 2.5^{\circ} \mathrm{C}, 12: 12 \mathrm{~L}: \mathrm{D}$, and $70.0 \% \pm 5.0 \% \mathrm{RH}$ when reared in water containing $0.5 \mathrm{\mu g}$ mycolactone/1 ml ethanol. $* \mathrm{ETH}=$ Ethanol; $\mathrm{ML}=$ Mycolactone.

mycolactone versus the blank $=-0.26 \pm 0.05$, Figure 5) or ethanol $\left(\mathrm{p}<0.0001 X^{2}=29.90\right.$, log odds of responding to ethanol versus the blank $=-0.27 \pm 0.05$, Figure 5).

\section{DISCUSSION}

The work presented here revealed mycolactone impacts larval development and survivorship of Ae. aegypti. Mycolactone impacted mosquito development from the egg to the pupal stage. Immature mosquitoes in the control groups needed approximately $7.2 \mathrm{~d}$ to reach the pupal stage, which is similar to results from a past study conducted under similar conditions (Christophers, 1960; Imam et al., 2014). However, those exposed to the high mycolactone dose needed approximately $15 \%$ more time to reach the pupal stage. Similarly, development from egg to adult exhibited a treatment effect (Table 1); however, this difference was never more than half a day and was most likely due to scheduled observations (e.g., observations every $12 \mathrm{~h}$ ) rather than biological significance. Additional studies with more refined observation periods should be conducted to determine whether the response is statistically different. Furthermore, fieldwork should be conducted to determine whether these data translate to natural populations.

In addition, the response trended by dose where the high and low doses reduced survivorship from egg to pupa and adult (Figure 2), and the middle dose appeared to be optimal. The survival of eggs to the pupal stage in the control groups was above $80 \%$, while those exposed to the high and low mycolactone dose were between 35 and 40\%. 


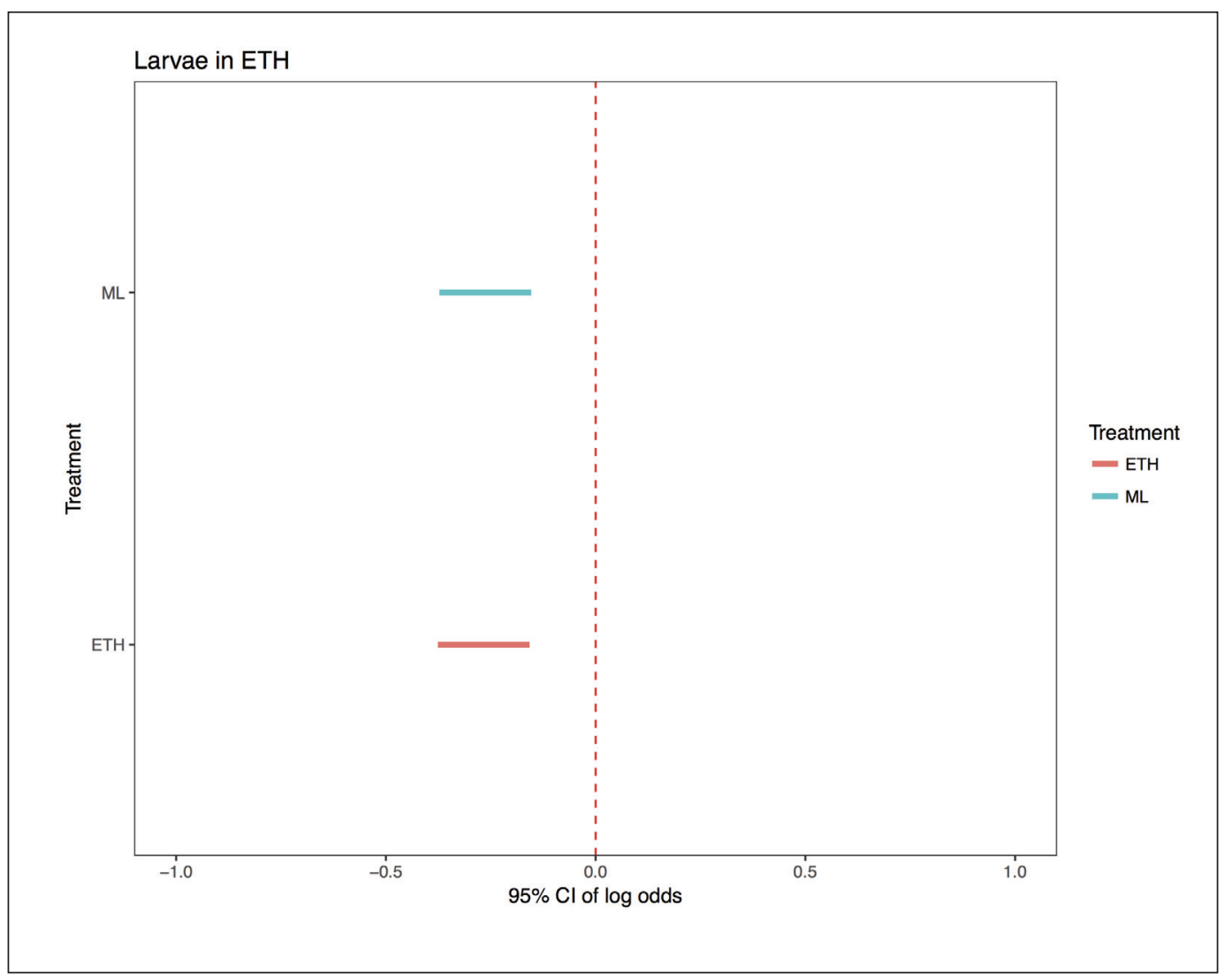

Figure 5. Log odds of Ae. aegypti laying eggs on filter paper containing $0.5 \mu \mathrm{g}$ mycolactone/1 ml ethanol at $25.0^{\circ} \mathrm{C} \pm 2.5^{\circ} \mathrm{C}, 12: 12 \mathrm{~L}: \mathrm{D}$, and $70.0 \% \pm 5.0 \% \mathrm{RH}$ when reared in water containing $1 \mathrm{ml}$ ethanol. $* \mathrm{ETH}=$ Ethanol; $\mathrm{ML}=$ Mycolactone.

In contrast, survivorship of eggs to the pupal stage from those exposed to the middle dose, while still lower than the control groups, had a $58 \%$ survivorship.

Throughout the course of these experiments, immature mosquitoes exposed to the middle mycolactone dose consistently produced greater survivor rates compared to other treatments, and yielded survivorship closest to the control group, possibly indicating a concentration window of suitability. These results are not surprising as such optima in mosquitoes have been recorded in response to a number of abiotic conditions. For example, too high temperatures can result in larval mortality, while too low temperatures stalled development (Couret et al., 2014). Specifically, room temperatures above $30^{\circ} \mathrm{C}$ resulted in larval mortality, while temperatures between $20-30^{\circ} \mathrm{C}$ resulted in optimal development and survivorship.

While not examined in this study, one explanation for the specific dose-response to mycolactone on mosquito larval development could relate to shifts in available nutrients. As previously indicated, $M$. ulcerans occurs in mostly lentic habitats where Ae. aegypti larvae also occur (Wallace et al., 2010; McIntosh et al., 2014). Bacteria in these habitats are known to breakdown organic matter, which then serves as a primary food substrate of the mosquito larvae (Kaufman et al., 1999). Recent research published by Souza et al. (2019) demonstrated shifts in microbial communities impact Ae. aegypti development time; more specifically, pupal development can be delayed significantly depending on which microbes were present during larval 
development (ranging from $7.5 \mathrm{~d}$ for the control to $18.4 \mathrm{~d}$ when larvae were presented with Escherichia coli (Souza et al., 2019).

The macrolide structure of mycolactone is similar to some QS molecules and has been hypothesized to function as a modulator of QS machinery or toward other mechanisms of secondary metabolism. Additionally, our group has found that mycolactone is a QS antagonist to some commensal and environmental bacteria. In the case of the present study, mycolactone present in the growth environments at certain concentrations could be inhibiting other microbes (i.e., bacteria) competing with $M$. ulcerans for similar resources. This inhibition could, in turn, be suppressing bacterial populations within the growth medium that are directly necessary for optimal larval mosquito development. Work is currently underway by our group for further examination with respect to $M$. ulcerans, and corresponding mycolactone, interactions with mosquitoes under natural, polymicrobial conditions.

Another possibility is that mycolactone could be impacting mosquito gut bacteria, which also play a crucial role in mosquito development. While not with our species, Chouaia et al. (2012) determined that Asaia symbiotic bacteria are beneficial in the development of immature Anopheles stephensi, (L.) (Diptera: Culicidae). Development of An. stephensi larvae reared in a habitat with rifampicin were delayed two to four days compared with those in the control (Chouaia et al., 2012). In the current study, mycolactone could have a similar impact; however, the associated bacterial community was not measured in this study. Future studies examining the effects of $M$. ulcerans and mycolactone on the microbial community associated with $A$. aegypti will shed critical light on our understanding of the mechanisms linking $M$. ulcerans and the yellow fever mosquito.

With regards to the behavioral study, our data indicate mosquitoes reared in the presence of mycolactone could be biased with regards to oviposition site selection. In this case, mosquitoes were more likely to deposit eggs in an environment containing mycolactone if they themselves were reared in such an environment. McCall and Eaton (2001) demonstrated adult Culex quinquefasciatus, Say (Diptera: Culicidae) reared in the presence of skatole (innately a repellent at high concentrations) or $p$-Cresole were more likely to deposit eggs in environments containing this compound. Accordingly, Sanford et al. (2012) determined similar "learning" abilities with adults being able to recognize and respond to these odors in anticipation of securing a food source, thus lending support the legacy effect hypothesis. In this case, volatile compounds potentially associated with microbes in the aquatic environment elicit the mosquito response. Others have demonstrated similar mosquito responses. For example, Ae. aegypti and Anopheles gambiae, Giles (Diptera: Culicidae) utilize indole (i.e., microbial byproduct) as a means for determining oviposition sites (Bohbot et al., 2011). Finally, as mentioned previously, gravid Ae. aegypti use volatiles in the form of carboxylic acids and methyl esters emitted from alpha and gamma proteobacteria as potent oviposition stimulants (Ponnusamy et al., 2008).

But why would mosquitoes in the current study deposit eggs in an environment not conducive for optimal larval development and survivorship? One explanation could be due to the mosquito population used in the current study are from a colony maintained without previous selective pressures by $M$. ulcerans, which could explain the partial morality we observed. Much like mosquitoes developing resistance or tolerance to insecticides, mosquitoes breeding in natural habitats with $M$. ulcerans could be undergoing the same, resulting in resident populations highly responsive to mycolactone, to locate hosts or oviposition sites.

A competing hypothesis is that the interaction between $M$. ulcerans and mosquitoes could be microbial manipulation of its host, whereby the microbe receives an advantage (i.e., distribution as previously discussed) and not the host. Such behavioral shifts are well documented for other microbes or parasites. For example, crickets infected with a parasite seek out aquatic habitats which result in their mortality but survivorship of the parasite (Hughes et al., 2012). 
Similarly, ants infected with a fungus exhibit similar behaviors resulting in enhance dispersal of the pathogen but to the detriment of the host (Hughes, 2013). Such a fascinating question beckons greater examination in future research as resulting data could provide insights into the evolutionary relationship between $M$. ulcerans, its toxin, and mosquito behavior. A potential scenario could be regions endemic with Ae. aegypti and $M$. ulcerans result in adult mosquitoes serving as a mechanism for distributing of the pathogen to new locations through subsequent oviposition events.

Results from the current study demonstrate a potential ecological link between M. ulcerans and Ae. aegypti in endemic environments where both species occur as well as other pathogens including yellow fever and dengue. Understanding this could prove crucial for deciphering the etiology of the pathogen and its mode of transmission. However, additional research is needed with viable $M$. ulcerans cells to determine whether mycolactone production, the production of other secondary metabolites or compounds, may impact mosquito development and oviposition site selection. If these results remain true, a synergism between the incidence of BU and yellow fever in non-immunized individuals could be possible and would require further investigation in these endemic areas. Furthermore, while we examined three concentrations of mycolactone encompassing concentrations detected in nature, future studies should explore multiple concentrations at a finer scale to determine the specific range eliciting the physiological and behavioral responses observed in the research presented in this manuscript. Additionally, determining the impact of mycolactone on other life-history traits (i.e., development over time) and morphometrics (e.g., size of adults) of mosquitoes is needed to better understand these interactions. Furthermore, while we verified UV- and biological activity, and retention factor (Rf) against a mycolactone control, it cannot be ruled out that minor mycolactone congeners or other minor lipids that ran within the same $\mathrm{Rf}$ value were also constituents of the purified mycolactone used in these experiments and might have affected mosquito development and behavior.

Acknowledgements. We would like to thank reviewers and Le Zheng for statistical analysis help. Also, the author would like to thank the government of Saudi Arabia, Cultural Mission of the Royal Embassy of Saudi Arabia in Washington (SACM) and Jazan University, College of Science, Saudi Arabia for providing financial support.

\section{Conflict of Interest}

The authors declare that they have no conflict of interest.

\section{REFERENCES}

Adusumilli, S., Mve Obiang, A., Sparer, T., Meyers, W., Hayman, J. \& Small, P.L.C. (2005). Mycobacterium ulcerans toxic macrolide, mycolactone modulates the host immune response and cellular location of $M$. ulcerans in vitro and in vivo. Cellular Microbiology 7(9): 12951304.

Albeny-Simões, D., Murrell, E.G., Elliot, S.L., Andrade, M.R., Lima, E., Juliano, S.A. \& Vilela, E.F. (2014). Attracted to the enemy: Aedes aegypti prefers oviposition sites with predator-killed conspecifics. Oecologia 175(2): 481-492.

Bohbot, J.D., Jones, P.L., Wang, G., Pitts, R.J., Pask, G.M. \& Zwiebel, L.J. (2011). Conservation of indole responsive odorant receptors in mosquitoes reveals an ancient olfactory trait. Chemical Senses 36(2): 149-160.

Chouaia, B., Rossi, P., Epis, S., Mosca, M., Ricci, I., Damiani, C. \& Favia, G. (2012). Delayed larval development in Anopheles mosquitoes deprived of Asaia bacterial symbionts. BMC Microbiology 12(1): 1-8.

Christophers, S.R. (1960). Aedes aegypti (L.). The Yellow Fever Mosquito. Its Life History, Bionomics and Structure. Rickard Christophers, Cambridge University Press, Cambridge, UK. 
Corbet, S. (1985). Insect chemosensory responses: A chemical legacy hypothesis. Ecological Entomology 10(2): 143-153.

Couret, J., Dotson, E., Benedict, M. \& Oliveira, P. (2014). Temperature, Larval Diet, and Density Effects on Development Rate and Survival of Aedes aegypti (Diptera: Culicidae). PloS One 9(2): e87468.

Garnham, P.C.C., Harper, J.O. \& Highton, R.B. (1946). The mosquitos of the Kaimosi Forest, Kenya colony, with special reference to yellow fever. Bulletin of Entomological Research 36(4): 473-496.

Hughes, D. (2013). Pathways to understanding the extended phenotype of parasites in their hosts. Journal of Experimental Biology 216(1): 142-147.

Hughes, D.P., Brodeur, J. \& Thomas, F. (2012). Host Manipulation by Parasites, Oxford University Press.

Imam, H., Zarnigar, G.S. \& Seikh, A. (2014). The basic rules and methods of mosquito rearing (Aedes aegypti). Tropical Parasitology 4(1): 53.

Kaufman, M.G., Walker, E.D., Smith, T.W., Merritt, R.W. \& Klug, M.J. (1999). Effects of larval mosquitoes (Aedes triseriatus) and stemflow on microbial community dynamics in container habitats. Applied and Environmental Microbiology 65(6): 2661-2673.

Marion, E., Prado, S., Cano, C., Babonneau, J., Ghamrawi, S. \& Marsollier, L. (2012). Photodegradation of the Mycobacterium ulcerans toxin, mycolactones: Considerations for handling and storage. PLoS One 7(4): e33600.

Marsollier, L., Robert, R., Aubry, J., Saint André, J.P., Kouakou, H., Legras, P. \& Carbonnelle, B. (2002). Aquatic insects as a vector for Mycobacterium ulcerans. Applied and Environmental Microbiology 68(9): 4623-4628.

McCall, P.J. \& Eaton, G. (2001). Olfactory memory in the mosquito Culex quinquefasciatus. Medical and Veterinary Entomology 15(2): 197-203.
McIntosh, M., Williamson, H., Benbow, M.E. Kimbirauskas, R., Quaye, C., Boakye, D., Small, P. \& Merritt, R. (2014). Associations between Mycobacterium ulcerans and aquatic plant communities of West Africa: Implications for Buruli ulcer disease. Ecohealth 11(2): 184-196.

Merritt, R.W., Walker, E.D., Small, P.L., Wallace, J.R., Johnson, P.D., Benbow, M.E. \& Boakye, D.A. (2010). Ecology and transmission of Buruli ulcer disease: A systematic review. PLoS Neglected Tropical Diseases 4(12): e911.

Miller, M.B. \& Bassler, B.L. (2001). Quorum sensing in bacteria. Annual Reviews in Microbiology 55(1): 165-199.

Mve-Obiang, A., Lee, R.E., Portaels, F. \& Small, P.L.C. (2003). Heterogeneity of mycolactones produced by clinical isolates of Mycobacterium ulcerans: Implications for virulence. Infection and Immunity 71(2): 774-783.

Ponnusamy, L., Xu, N., Nojima, S., Wesson, D.M., Schal, C. \& Apperson, C.S. (2008). Identification of bacteria and bacteriaassociated chemical cues that mediate oviposition site preferences by Aedes aegypti. Proceedings of the National Academy of Sciences 105(27): 92629267.

Quek, T.Y., Athan, E., Henry, M.J., Pasco, J.A., Redden-Hoare, J., Hughes, A. \& Johnson, P.D. (2007). Risk factors for Mycobacterium ulcerans infection, southeastern Australia. Emerging Infectious Diseases 13(11): 1661-1666.

Romero, D., Traxler, M.F., López, D. \& Kolter, R. (2011). Antibiotics as signal molecules. Chemical Reviews 111(9): 5492-5505.

Sanders, M., Jordan, H., SerewisPond, C., Zheng, L., Benbow, M., Small, P. \& Tomberlin, J. (2017). Mycobacterium ulcerans toxin, Mycolactone May Enhance HostSeeking and Oviposition Behavior by Aedes aegypti (L.)(Diptera: Culicidae). Environmental Microbiology 19(5): 1750 -1760. 
Sanford, M.R. \& Tomberlin, J.K. (2011). Conditioning individual mosquitoes to an odor: Sex, source, and time. PloS One 6(8): e24218.

Sanford, M.R., Olson, J.K., Lewis, W.J. \& Tomberlin, J.K. (2012). The effect of sucrose concentration on olfactorybased associative learning in Culex quinquefasciatus Say (Diptera: Culicidae). Journal of Insect Behavior 26(4): 494-513.

Souza, R.S., Virginio, F., Riback, T.I.S., Suesdek, L., Barufi, J.B. \& Genta, F.A. (2019). Microorganism-based larval diets affect mosquito development, size and nutritional reserves in the yellow fever mosquito Aedes aegypti (Diptera: Culicidae). Frontiers in Physiology 10: 152.

Tomberlin, J.K., Crippen, T.L., Wu, G., Griffin, A.S., Wood, T.K. \& Kilner, R.M. (2016). Indole: An evolutionarily conserved influencer of behavior across kingdoms. Bioessays 39(2): 1600203.

Vincent, Q.B., Ardant, M.-F., Adeye, A., Goundote, A., Saint-André, J.-P., Cottin, J., Kempf, M., Agossadou, D., Johnson, C. \& Abel, L. (2014). Clinical epidemiology of laboratory-confirmed Buruli ulcer in Benin: A cohort study. The Lancet Global Health 2(7): e422-e430.

Wallace, J., Gordon, M., Hartsell, L., Mosi, L., Benbow, M.E., Merritt, R. \& Small, P.L.C. (2010). Interaction of Mycobacterium ulcerans with mosquito species: Implications for transmission and trophic relationships. Applied and Environmental Microbiology 76(18): 6215-6222.

Wallace, J.R., Mangas, K.M., Porter, J.L., Marcsisin, R., Pidot, S.J., Howden, B.O., Omansen, T.F., Zeng, W., Axford, J.K. \& Johnson, P.D. (2017). Mycobacterium ulcerans low infectious dose and atypical mechanical transmission support insect bites and puncturing injuries in the spread of Buruli ulcer. Plos Neglected Tropical Diseases 11(4): e0005553.
Williamson, H.R., Mosi, L., Donnell, R., Aqqad, M., Merritt, R.W. \& Small, P.L. (2014). Mycobacterium ulcerans fails to infect through skin abrasions in a guinea pig infection model: Implications for transmission. PLoS Neglected Tropical Diseases 8(4): e2770.

Williamson, H.R., Benbow, M.E., Campbell, L.P., Johnson, C.R., Sopoh, G., Barogui, Y., Merritt, R.W. \& Small, P.L. (2012). Detection of Mycobacterium ulcerans in the environment predicts prevalence of Buruli ulcer in Benin. PLoS Neglected Tropical Diseases 6(1): e1506.

Williamson, H.R., Benbow, M.E., Nguyen, K.D., Beachboard, D.C., Kimbirauskas, R.K., McIntosh, M.D. \& Small, P.L. (2008). Distribution of Mycobacterium ulcerans in Buruli ulcer endemic and nonendemic aquatic sites in Ghana. PLoS Neglected Tropical Diseases 2(3): e205.

Yeboah-Manu, D., Kpeli, G.S., Ruf, M.T., Asan-Ampah, K., Quenin-Fosu, K., Owusu-Mireku, E. \& Newman, M. (2013). Secondary bacterial infections of buruli ulcer lesions before and after chemotherapy with streptomycin and rifampicin. PLoS Neglected Tropical Diseases 7(5): e2191.

Zhang, X., Crippen, T.L., Coates, C.J., Wood, T.K. \& Tomberlin, J.K. (2015). Effect of quorum sensing by Staphylococcus epidermidis on the attraction response of female adult yellow fever mosquitoes, Aedes aegypti aegypti (Linnaeus) (Diptera: Culicidae), to a blood-feeding source. PLoS One 10: e0143950. 